\title{
Experimental and Numerical Study of Capillary Driven Flow in Vane-type Tank under Normal Gravity and Microgravity Conditions
}

\author{
Ning Weng ${ }^{1}$, Qinggong Wang ${ }^{1}$, Yuying Wang ${ }^{2}$, Jinyin Huang ${ }^{2}$, Letian Yao², Wei Yao ${ }^{1}$, Jindong Li ${ }^{2}$ \\ ${ }^{1}$ Qian Xuesen Laboratory of Space Technology/China Academy of Space Technology \\ No. 104 Youyi Road, Haidian District, Beijing, China \\ 416930025@qq.com; wqg1804@163.com; yaowei@qxslab.cn \\ ${ }^{2}$ Institute of Spacecraft System Engineering / China Academy of Space Technology \\ No. 104, Youyi Road, Beijing, China \\ wangyuying@buaa.edu.cn; huang_jinyin@163.com; 504108260@qq.com; ljdcast@163.com
}

\section{Extended Abstract}

Capillary flow of the low surface tension liquid in interior corners between two intersecting surfaces and/or in containers have received much attention in recent years. It relates to a wide range of applications in the fields of microfluidics, fluid management in space and microgravity, porous media, etc. To optimise the design and efficiency of reservoir applications which involve capillary flow in both normal gravity and microgravity, it is necessary to have a basic understanding of the involved flow physics. Particularly, when cryogenic liquids are used, such as the liquid hydrogen, liquid nitrogen, liquid neon and liquid helium, they show quite low surface tension and a low temperature out of the scope of current understanding on capillary flows.

In the present study, a combined experimental and numerical approach is employed to study the capillary flow dynamics of a liquid subject to the different gravity levels. First, we perform experiments both on earth and in a $3.6 \mathrm{~s}$ drop tower. A normal working liquid of ethanol is used in the tests since it is somewhat hard to adopt the cryogenic liquid directly when it requires a strict control on the working temperature in the measurement. The experimental results show the transient development of the fluid in capillary structures, and it well meets the Concus-Finn condition. We use the results for model validation for the numerical studies.

For numerical studies, computational fluid dynamics (CFD) models are developed using the Volume of Fluid (VOF) [1] and Continuous Surface Force (CSF) [2] method. A Kistler dynamic contact angle model [3] is used as the boundary condition at the three-phase moving contact line, which considers the effects of the contact angle hysteresis. An adaptive mesh refinement (AMR) is applied to refine the liquid-gas interface for higher accuracy. The dependence of numerical results on the mesh refinement is first examined, and the difference in the predictions of the hight of capillary rise of liquid is less than 5\%. Then, the liquid flow in the corner is the investigated numerically in both normal gravity and microgravity. The experimental result and numerical result are basically consistent. It is certified that the numerical method with the dynamic contact angle model are able to predict accurately the dynamics of the capillary flow in both normal gravity and microgravity. After validation, we extend the numerical simulations for different cryogenic liquids, and examine the main operating parameters, including the capillary structure, number of vanes, and initial wettability of fluid. The results would provide some guideline for the design of the low surface tension vane-type vane tank for space applications.

\section{References}

[1] J. B. Lee, D. Derome, A. Dolatabadi, J. Carmeliet, "Energy budget of liquid drop impact at maximum spreading: numerical simulations and experiments", Langmuir. Vol. 32, pp. 1279-1288, 2016.

[2] J. Brackbil, D. Kothe, C. Zemach, "A continuum method for modelling surface tension”, J. Comput. Phys. vol. 100 no. 2, pp. 335-354, 1992.

[3] S. F. Kistler, "Hydrodynamics of wetting," in Wettability, J. C. Bergs, Ed. New York: Marcel Dekker, 1993 , pp. 311. 